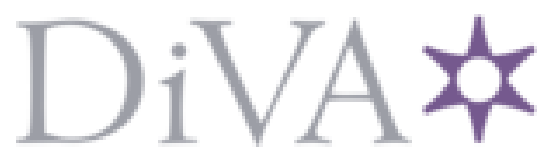

http://www.diva-portal.org

This is the published version of a paper presented at 2019 IEEE 32nd International Conference on Micro Electro Mechanical Systems (MEMS), 27-31 Jan. 2019.

Citation for the original published paper:

Pagliano, S., Gota, F., Raja, S N., Dubois, V J., Stemme, G. et al. (2019)

Feedback-Free Electromigrated Tunneling Junctions from Crack-Defined Gold

Nanowires

In: Proceedings of the IEEE International Conference on Micro Electro Mechanical Systems (MEMS) (pp. 365-367). IEEE conference proceedings https://doi.org/10.1109/MEMSYS.2019.8870698

N.B. When citing this work, cite the original published paper.

Permanent link to this version:

http://urn.kb.se/resolve?urn=urn:nbn:se:kth:diva-268311 


\title{
FEEDBACK-FREE ELECTROMIGRATED TUNNELING JUNCTIONS FROM CRACK-DEFINED GOLD NANOWIRES
}

\author{
Simone Pagliano ${ }^{l}$, Fabrizio Gota ${ }^{l}$, Shyamprasad N. Raja ${ }^{l}$, Valentin Dubois ${ }^{l}$, \\ Göran Stemme ${ }^{1}$ and Frank Niklaus ${ }^{1}$ \\ KTH Royal Institute of Technology, Stockholm, SWEDEN
}

\begin{abstract}
Tunneling junctions are pairs of electrodes separated by gaps of a few nanometers $(<3 \mathrm{~nm})$ that allow electrons to tunnel across the gap. Tunneling junctions are of great importance for applications such as label-free biomolecule sensing and single molecule electronics, but their fabrication remains difficult and laborious. In this paper, we present a simple 2-stage process for the fabrication of tunneling junctions consisting of electrode pairs made of gold (Au). This is achieved by combining a novel methodology for fabricating crack-defined Au nanowires at wafer-scale with a constant voltage, feedback-free electromigration procedure to form tunneling nanogaps free of debris.
\end{abstract}

\section{INTRODUCTION}

Tunneling nanogaps are being explored today for numerous applications, such as molecular electronics[1], bio-molecule detection [2], nano-photonics [3], and DNA sequencing [4]. The lack of parallel fabrication processes with high yields for realizing tunneling nanogaps is however hindering the development of both new research tracks and market applications.

Mechanically-Controlled-Break-Junctions (MCBJs) [5] and Electromigrated-Break-Junctions (EBJs) [6] are the most common forms of Au tunneling junctions used today, particularly in the field of molecular electronics. In MCBJs, the nanogap is created by mechanically pulling apart the two extremities of an $\mathrm{Au}$ nanowire using a piezoelectric stage until the nanowire breaks. In EBJs, the nanogap is created by forcing a high current density through a neck-shaped nanowire, such that $\mathrm{Au}$ atoms are moved away from the neck by the wind force of the flowing electrons. During the process, both techniques require high-speed monitoring of the decrease of conductance of each nanowire and active feedback systems to adapt the applied stimulus to avoid catastrophic failure. Although both techniques allow the fabrication of sub- $2 \mathrm{~nm}$ gaps with high reliability, the requirement of feedback-systems prevents the conversion of these methodologies into scalable fabrication platforms. While simultaneous electromigration of a small number of parallel nanowires $(\sim 8)$ has been demonstrated without the use of a feedback system [7], the nanowires were patterned by e-beam lithography, which is a serial fabrication process.

Other parallel fabrication processes, based on shadow evaporation [8] or lateral expansion of easily oxidized metals [9] have been proposed. However, these processes do not allow the generation of the atomically thin tips that are necessary for molecular electronics and DNA sequencing. Recently, an alternative method compatible with wafer-scale processes called the Crack-DefinedBreak-Junction (CDBJ) methodology has been reported by our group [10]. With this method a yield of $7 \%$ for producing sub-3nm gaps at very high device density was achieved. It was also discovered that a much higher yield of devices with one or more $\mathrm{Au}$ nanowires could be produced by design using the CDBJ methodology

In this work, we present a novel methodology that combines the wafer-scale fabrication capability of the CDBJ methodology for $\mathrm{Au}$ nanowires, with a simple constant-voltage electromigration procedure to fabricate tunneling junctions.

\section{METHODOLOGY}

The fabrication process for realizing our tunneling junctions consists of two stages. In the first stage, $\mathrm{Au}$ nanowires were manufactured by simultaneously stretching thousands of beams of ductile conductive material $(\mathrm{Au})$ at wafer-scale. In the second stage, tunneling junctions were formed from the nanowires using a feedback-free electromigration procedure at constant voltage.

In the first stage, we deposited a layer-stack composed of $50 \mathrm{~nm}$ of titanium nitride (TiN), 3nm of $\mathrm{Cr}$ as the adhesion layer and $10 \mathrm{~nm}$ of $\mathrm{Au}$ on top of a $200 \mathrm{~nm}$ thick sacrificial layer of amorphous silicon (a-Si), as in Fig.1a. The TiN was deposited by atomic layer deposition at a temperature of $350{ }^{\circ} \mathrm{C}$, producing residual tensile stress in this layer at room temperature. Using i-line projection lithography, photoresist was patterned into necked bridges on top of the layer-stack. Although the nominal resolution of our i-line photo-lithographic tool is $\sim 500 \mathrm{~nm}$, we obtained necked bridges that were between $90 \mathrm{~nm}$ and 250 nm wide at the neck using mask designs of overlapping triangles in combination with over-exposure of the resist. a)

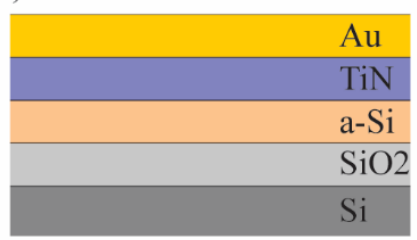

c)

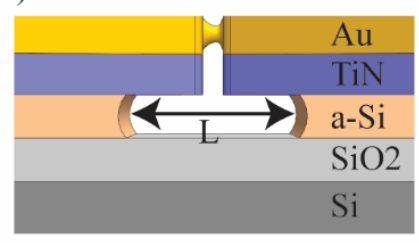

b)

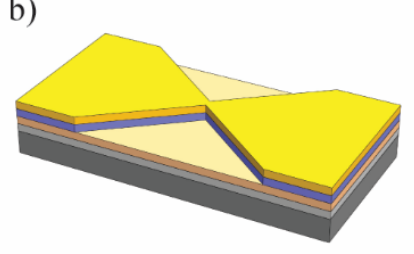

d)

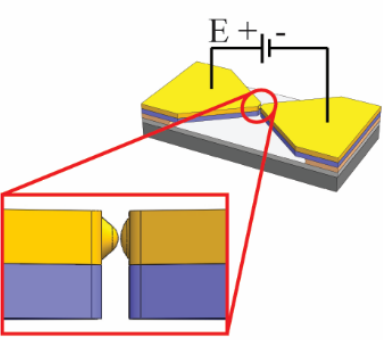

Figure 1: Schematic of fabrication process. (a)Crosssection of deposited layers. (b)Necked beam of Au and TiN (c) Au nanowire forms at the neck as a result of TiN fracture induced by etching of a-Si. The width of the gap depends on the bridge length (d) Au nanogap formed by electromigration of an Au nanowire 

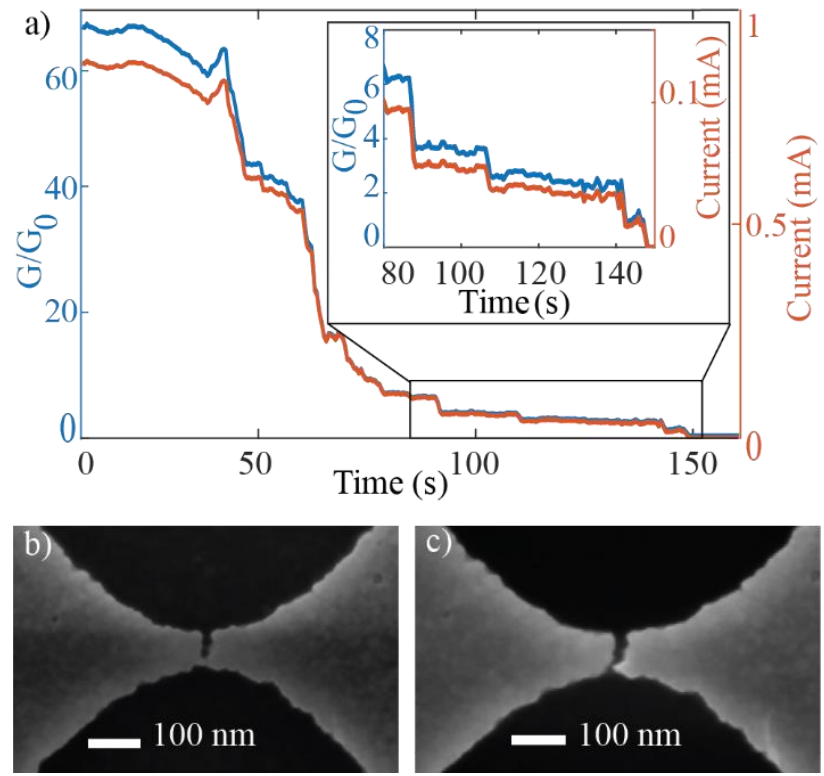

Figure 2: Electromigration. (a) SEM picture of a junction with nanowires before electromigration; (b) SEM picture of the same junction after electromigration; (c) Decrease of the conductance of a typical junction over time during electromigration. A zoom on the last part of the curve, before electromigration, is presented in the inset

The patterns were then transferred to the layer-stack by a combination of ion beam milling and reactive ion etching (Fig. 1b). The a-Si was then removed by isotropic dry silicon etching with $\mathrm{SF}_{6}$. Upon removal of a-Si, the brittle TiN bridges fractured at the constriction due to the residual tensile stress in the TiN layer. The two TiN sections then receded, pulling apart the $\mathrm{Au}$ overlying the crack-site, which deformed to create voids and low aspect-ratio nanometric ligaments, referred to as nanowires in this work (Fig.1c).

In the second stage, electromigration was performed on these nanowires in a probe station at room temperature in open atmosphere, by applying a constant voltage of 230 $\mathrm{mV}$ (Fig. 1d). The process was stopped either after the conductance of the nanowires fell below $0.01 * \mathrm{G}_{0}$, where $\mathrm{G}_{0}=77.48 \mu \mathrm{S}$ is the quantum of conductance, or after a time of 10 minutes had elapsed.

\section{RESULTS}

The formation of nanowires in the Au layer depends on the pulling action of the two retracting TiN cantilevers sections after crack formation, and the plastic deformation of the ductile $\mathrm{Au}$. Since the force applied by the TiN sections on the Au layer depends on the bridge length and the tensile stress in the TiN film, it can be reliably adjusted and reproduced. In contrast, plastic deformation of $\mathrm{Au}$ is stochastic, as it is dominated by shear stresses and influenced by the polycrystallinity of the TiN and the Au. SEM imaging showed that nanowires can be formed on bridges with lengths ranging from $1.5 \mu \mathrm{m}$ up to $6 \mu \mathrm{m}$. We also observed that the width of the bridge constrictions has a strong influence on the number of nanowires formed in the gap. While no more than two nanowires are formed in
$90 \mathrm{~nm}$ wide necks, more than four nanowires can be formed in $250 \mathrm{~nm}$ wide constrictions.

The resistance of $51 \quad 3.3 \mu \mathrm{m}$-long bridges was measured before electromigration, by applying a low voltage of $50 \mathrm{mV}$, so as to not induce any structural modification to the nanowires. This revealed that 36 of the 51 gaps $(72 \%)$ showed ohmic behavior indicating the presence of $\mathrm{Au}$ nanowires, with resistances ranging from $90 \Omega$ to $1.5 \mathrm{k} \Omega$, or equivalently from 143 to 8 times the conductance quantum $\mathrm{G}_{0}$. The remaining 15 devices did not show a measurable current, and were assumed not to contain any nanowires bridging the crack. A histogram of the normalized conductance of the nanowires, $\mathrm{G} / \mathrm{G}_{0}$, is shown in Fig. 4 a.

We performed electromigration on the ohmic devices only. During electromigration, the conductance fell below the established threshold of $0.01 * \mathrm{G}_{0}$ in 21 devices. An example is shown in Fig. 2b. In 10 of these junctions, electromigration occurred with a steep decrease of the conductance from the initial value to below the threshold in less than 10 seconds. In the remaining devices, we observed a gradual decrease of conductance from high $\mathrm{G} / \mathrm{G}_{0}$, suggesting that the cross-section of the nanowires was shrinking, and the electron transport was still ohmic, as shown in Fig. 2c. At values of $\mathrm{G} / \mathrm{G}_{0}$ below 10 , the decrease in conductance occurred in discrete steps, suggesting that electrical transport through the junction was ballistic, as shown in the inset of Fig. 2c. SEM images of devices before and after electromigration are shown in Fig. $2 \mathrm{a}$ and $2 \mathrm{~b}$ respectively.

We applied a voltage sweep from 0 to $100 \mathrm{mV}$ to measure the I-V characteristics of the junctions immediately after electromigration. Tunneling currents were measured in 19 of them, corresponding to $53 \%$ of the ohmic devices and $37 \%$ of the entire pool of tested devices. As shown in Fig. 3, tunneling junctions show linear I-V curves at low voltages.

A tunneling resistance can therefore be calculated by applying a linear fit to the I-V curves. We obtained tunneling resistances between $3 \mathrm{M} \Omega$ and $240 \mathrm{G} \Omega$ as shown in Fig. 4 a.

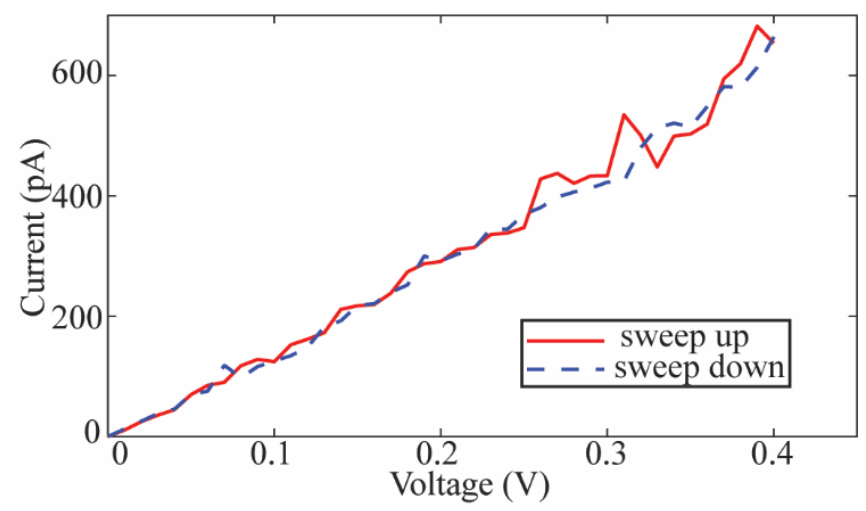

Figure 3: Electrical characterization. Example of an I-V curve of an electromigrated junction. The measured I-V curves are typically linear up to an applied bias of $\sim 0.3 \mathrm{~V}$, and becomes non-linear for higher voltages. The tunneling resistance of the junction presented here is $170 \mathrm{M} \Omega$. 

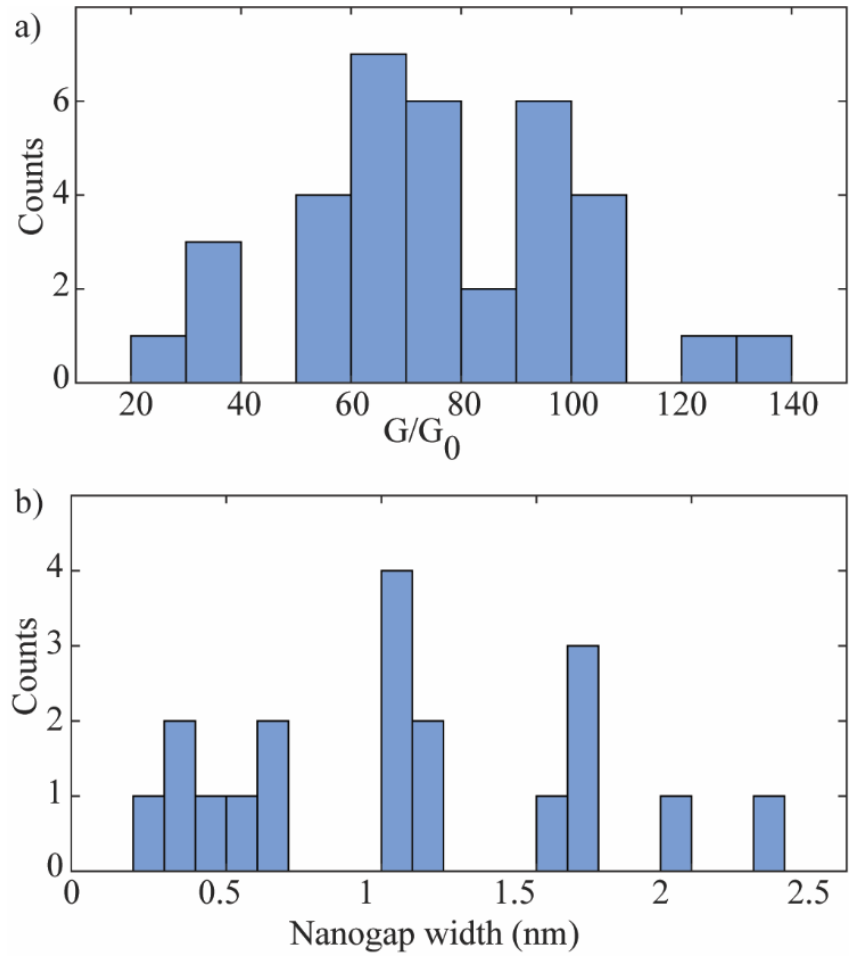

Figure 4: Statistics of Device Characteristics. (a) Histogram of the normalized conductance $\mathrm{G} / \mathrm{G}_{0}$ of nanowires before electromigration, (b) Histogram of gap width for tunneling junctions

The measured I-V curves were also fitted with a 1-D transport model across symmetric barriers[10],[11] to extract the width of the tunneling devices. It was found that the junctions featured nanogap widths between 0.3 and 2.2 nm (Fig.4b).

\section{CONCLUSIONS}

We have fabricated and electrically characterized $\mathrm{Au}$ tunneling junctions using a novel two-stage manufacturing process. Our methodology combines parallel fabrication of Au nanowires in the wafer scale with on-demand formation of nanogaps free of debris using a constant voltage electromigration process. Although the electromigration is performed serially, the simplicity of feedback free electromigration offers the potential for parallelization.

\section{ACKNOWLEDGMENT}

This work was supported by the Swedish Research Council (Grant No.2016-04852) and the European Research Council (Grant No. 277879).

\section{REFERENCES}

[1] M. Di Ventra and M. Taniguchi, "Decoding DNA, RNA and peptides with quantum tunnelling," Nat. Nanotechnol., vol. 11, no. 2, pp. 117-126, Feb. 2016.

[2] F. Schwarz and E. Lörtscher, "Breakjunctions for investigating transport at the molecular scale," J. Phys. Condens. Matter, vol. 26, no. 47, p. 474201, Nov. 2014.

[3] W. Kubo and S. Fujikawa, "Au Double Nanopillars with Nanogap for Plasmonic Sensor," 2011.

[4] P. Pang et al., "Fixed-Gap Tunnel Junction for Reading DNA Nucleotides," 2014.

[5] D. Xiang, H. Jeong, T. Lee, and D. Mayer, "Mechanically Controllable Break Junctions for Molecular Electronics," Adv. Mater., vol. 25, no. 35, pp. 4845-4867, Sep. 2013.

[6] H. Park, A. K. L. Lim, A. P. Alivisatos, J. Park, and P. L. McEuen, "Fabrication of metallic electrodes with nanometer separation by electromigration," Appl. Phys. Lett., vol. 75, no. 2, p. 301, Jul. 1999.

[7] H. Zhang, C. V. Thompson, F. Stellacci, and J. T. L. Thong, "Parallel fabrication of polymer-protected nanogaps," Nanotechnology, vol. 21, no. 38, 2010.

[8] L. F. Sun, S. N. Chin, E. Marx, K. S. Curtis, N. C. Greenham, and C. J. B. Ford, "Shadow-evaporated nanometre-sized gaps and their use in electrical studies of nanocrystals," Nanotechnology, vol. 16, no. 6, pp. 631-634, 2005.

[9] A. Fursina, S. Lee, R. G. S. Sofin, I. V. Shvets, and D. Natelson, "Nanogaps with very large aspect ratios for electrical measurements," Appl. Phys. Lett., vol. 92, no. 11, pp. 1-4, 2008.

[10] V. Dubois et al., "Massively parallel fabrication of crack-defined gold break junctions featuring sub-3 nm gaps for molecular devices," Nat. Commun., vol. 9, no. 1, pp. 1-10, 2018.

[11] A. Mangin, A. Anthore, M. L. Della Rocca, E. Boulat, and P. Lafarge, "Reduced work functions in gold electromigrated nanogaps," Phys. Rev. B, vol. 80, no. 23, p. 235432, Dec. 2009.

\section{CONTACT}

Simone Pagliano, simone.pagliano@eecs.kth.se 